\title{
Management of neuroblastoma: a study of first- and second-line chemotherapy responses, a single institution experience
}

\author{
Emmad E. Habib, Amr T. El-Kashef, Ezzat S. Fahmy \\ Clinical Oncology Department, Faculty of Medicine, Cairo University, Egypt
}

\begin{abstract}
Neuroblastoma is a high-grade malignancy of childhood. It is chemo- and radio-sensitive but prone to relapse after initial remission.

The aim of the current study was to study the results of the first- and second-line chemotherapy on the short-term response and long-term survival of children, and to further describe the side effects of treatment. Ninety-five children with advanced neuroblastoma were included in the study, divided into two groups according to the treatment strategy: 65 were treated by first-line chemotherapy alone, and 30 children who were not responding or relapsed after first-line chemotherapy were treated by second-line chemotherapy. External beam radiotherapy was given to bone and brain secondary cancers when detected. Staging workup was performed before, during and after management. Response was documented after surgery for the primary tumor. Median follow up was 32 months (range 24-60 months). Chemotherapy was continued until toxicity or disease progression occurred, indicating interruption of chemotherapy. Patients received a maximum of 8 cycles. Toxicity was mainly myelo-suppression, with grade II-III severity in $60 \%$ of the firstline and $70 \%$ of the second-line chemotherapy patients. Median total actuarial survival was nearly 51 months for the first-line chemotherapy group and 30 months for the second-line line group, with a statistically significant difference between the two groups $(\mathrm{P}<0.01)$.
\end{abstract}

\section{Introduction}

Neuroblastoma is the most common extra-cranial solid tumor in childhood; over $75 \%$ of patients are in an advanced stage at diagnosis and prognosis is poor. It is chemotherapy sensitive; $70 \%$ of patients

Correspondence: Emmad E. Habib, Clinical Oncology Department, Faculty of Medicine, Cairo University, Egypt. E-mail: cairo.oncology@gmail.com

Key words: chemotherapy, pediatric, neuroblastoma, survival.

Received for publication: 10 December 2011.

Revision received: 20 January 2012.

Accepted for publication: 3 February 2012.

This work is licensed under a Creative Commons Attribution NonCommercial 3.0 License (CC BY-NC 3.0).

CCopyright E.E. Habib et al., 2012

Licensee PAGEPress, Italy

Oncology Reviews 2012; 6:e3

doi:10.4081/oncol.2012.e3 with high-risk neuroblastoma could achieve complete remission after comprehensive therapy, including chemotherapy, surgery, radiotherapy, bone marrow transplantation, and biotherapy. However, the remission time interval is short, and the majority of patients die of tumor relapse. The 5-year survival of patients with high-risk NB is only approximately $30 \% .{ }^{1}$ At diagnosis, the defining characteristics of highrisk neuroblastoma include age over 1.5 years, advanced stage, amplification of the N-MYC oncogene, and histological findings. ${ }^{2-4}$

Progress in the treatment of high-risk neuroblastoma may have been due to the use of higher doses of chemotherapy ${ }^{1}$ and improved supportive care. Moreover, vitamin A (retinol) plays a critical role in normal neural crest development. Intracellular retinol is metabolized to all-trans retinoic acid (ATRA). Exposure of human neuroblastoma cell lines to supraphysiological doses of ATRA caused a reduction in cell growth and induction of neurite differentiation that was similar to normal neural cells. This property of vitamin A directed oncologists to use fenretinide, a vitamin A derivative, in the treatment of pediatric neuroblastoma in combination with chemotherapy. ${ }^{5,6}$ Further still, a coumarin derivative RKS262 belongs to a new class of potential antitumor agents. RKS262 was identified by structural optimization of nifurtimox, which is currently undergoing phase II clinical trials to treat high-risk neuroblastoma. ${ }^{7,8}$ In another attempt to improve the results of treatment of high-risk neuroblastoma cases, patients have received metronomic chemotherapy. Recent experimental studies have suggested that frequent administration of certain cytotoxic agents at low doses, known as metronomic chemotherapy, increases the anti-angiogenic activity of certain drugs. The advantage of this strategy is lower toxicity and risk of emergence of drug-resistant tumor cells than conventional administration. ${ }^{9-11}$ Monoclonal antibody therapy for neuroblastoma is also an attractive investigational treatment option. GD2-disialoganglioside is expressed on the surface of childhood neuroblastoma cells. Because of this tumor selective expression, it is an attractive target for tumor specific therapy with monoclonal antibodies. Over the last two decades, several anti-GD2 antibodies have been developed and investigated for the therapy and consolidation of pediatric neuroblastoma. ${ }^{12-14}$ For patients presenting with a large tumor burden at the time of treatment, 131-iodine-mIBG therapy is usually recommended. ${ }^{15}$ The aim of the current study was to study the outcome of first- and second-line conventional chemotherapy on the short-term response, and long-term survival of high-risk neuroblastoma cases.

\section{Materials and Methods}

Between June 2005 and December 2009, 95 neuroblastoma patients were diagnosed at the Pediatric Unit of the Oncology Department, Faculty of Medicine, Cairo University, Egypt. The median follow-up period was 32 months (range 24-60 months). Cases were either children 
with Evans stage III or IV who were newly diagnosed and treated with chemotherapy alone (65 cases) or those who failed initial successful therapeutic modalities (30 cases). The patients were younger than 16 years with a Karnovsky performance status of more than $30 \%$. Patients with a lower performance status were excluded from the study due to the lowered tolerance to chemotherapy. Whereas, with treatment, an improvement in performance status would be expected in responders.Sixty-five children were included in the first-line chemotherapy group alone and all were treated with 6-8 cycles of alternating courses of OPEC/OJEC ${ }^{16}$ every three weeks as follows: vincristine $1.5 \mathrm{mg} / \mathrm{m}^{2}$ Day 1, etoposide $200 \mathrm{mg} / \mathrm{m}^{2}$ Day 1, cyclophosphamide $600 \mathrm{mg} / \mathrm{m}^{2}$ Day 1 , and cisplatin $80 \mathrm{mg} / \mathrm{m}^{2}$ Day 1 or carboplatin $500 \mathrm{mg} / \mathrm{m}^{2}$ Day 1, these treatments alternating with each other every 21 days if there was hematologic recovery. Second-line therapy was given to 30 children and all were treated with 6-8 cycles according to the French SFOP studies protocol. ${ }^{17}$ This consisted of alternating courses of CAdO (cyclophosphamide 300 $\mathrm{mg} / \mathrm{m}^{2}$ Day 1 , vincristine $1.5 \mathrm{mg} / \mathrm{m}^{2}$ Day 1 , and doxorubicin $60 \mathrm{mg} / \mathrm{m}^{2}$ Day 1) and CE (carboplatin $40 \mathrm{mg} / \mathrm{m}^{2}$ Day 1-5 and etoposide $100 \mathrm{mg} / \mathrm{m}^{2}$ Day 1-5 every three weeks). All children were subjected to the same chemotherapy (C/T) for 6-8 cycles till remission, grade 3 or 4 toxicity, or disease progression occurred. Radiological evaluation of response to therapy was performed every 2 cycles before administration of the forthcoming cycle. External beam radiotherapy was given to bone and brain secondary cancers when detected irrespective of group.

\section{Diagnostic work up}

- A full clinical examination with documentation of all measurable disease.

- Performance status using K.I and body weight in kgs.

- Laboratory investigations included: CBC, BUN, serum creatinine, LDH, ESR, and urinary VMA.

- Bone marrow aspiration and tumor biopsy.

- Radiological imaging included: chest X-ray, CT scan of the tumor site and abdomino-pelvic sonography.

- Radionuclide imaging included: bone scan and 131I-MIBG scans.

Response criteria were as follows: ${ }^{18}$

- Complete response (CR): complete disappearance of disease.

- Partial response (PR): 50-90\% decrease in tumor volume.

- Stationary disease (SD): $<50 \%$ decrease in tumor volume.

- No response (NR): no change in size of tumor.

- Disease progression (DP): increase in size of tumor.

\section{Statistical analysis}

Independent proportions were compared using $\chi^{2}$ and Fisher's exact tests. Total actuarial survival curves were plotted with log rank test for survival time and ANOVA was used to compare groups. $\mathrm{P}<0.05$ was considered statistically significant.

\section{Results}

Patients' characteristics are summarized in Table 1. In the first-line chemotherapy group, bone marrow involvement was found in 39 cases (60\%) and lymph node metastases in $30(46.2 \%)$ of those 39 . Of those cases with bone marrow involvement, 38 of the 39 also had a positive bone scan and the remaining patient also had secondary brain tumors. Two cases with bone marrow and bone involvement also had soft tissue extension. In the second-line chemotherapy group, lymph node metastases were found in 15 cases (50\%), and all of those cases also had bone marrow involvement. Eleven of those 15 also had also a positive bone scan, 7 had secondary brain tumors, and the remaining 4 had soft tissue extension. Both performance status and body weight improved significantly after therapy (Figures 1 and 2, respectively). Mean Karnovsky Index \pm SD was $66.2 \% \pm 7.2 \%$ and $68.9 \% \pm 6.5 \%$ pre-therapy, and increased significantly to $84.9 \% \pm 9.73 \%$ and $82.4 \% \pm 4.1 \%$ post therapy in the first-line and second-line chemotherapy groups, respectively. The increase in the body weight in the groups was statistically significant ( $\mathrm{P}=3.72 \mathrm{e}-9$ and $\mathrm{P}=8.63 \mathrm{e}-15$, respectively).

\section{Short-term response assessment}

Response in the primary tumors is summarized in Table 2. Outcome of metastatic cases at presentation is summarized in Table 3. The toxicity of each treatment group is summarized in Table 4.

\section{Long-term survival assessment}

Figure 3 shows the total actuarial 5-year survival of the studied groups during the follow-up period. Median survival was 51 months for the first-line chemotherapy group and 33 months for the second-line chemotherapy group $(\mathrm{P}<0.01$ for both groups). At the end of the followup period (median follow-up 32 months, range 2-5 years), $40 \%$ of patients in the first-line chemotherapy group and $14 \%$ in the secondline chemotherapy group were still alive $(\mathrm{P}<0.01)$.

\section{Discussion}

Prognosis for high-risk neuroblastoma patients has improved over the last decades. However, even after highly intensive treatment, only

Table 1. The main clinical features of cases.

\begin{tabular}{|c|c|c|c|c|}
\hline \multirow{2}{*}{$\begin{array}{l}\text { Age range (years) } \\
\text { Median age } \\
\text { Males:females } \\
\text { Sites of disease }\end{array}$} & \multicolumn{2}{|c|}{$\begin{array}{c}\text { First-line } \\
\text { chemotherapy } \\
(\mathrm{n}=65) \\
1-13 \\
5 \\
3: 2\end{array}$} & \multicolumn{2}{|c|}{$\begin{array}{l}\text { Second-sine } \\
\text { chemotherapy } \\
\begin{array}{c}(\mathrm{n}=30) \\
6-15 \\
7 \\
2: 1\end{array}\end{array}$} \\
\hline & N. & $\%$ & N. & $\%$ \\
\hline \multicolumn{5}{|l|}{ Primary tumor } \\
\hline Thoracic & 18 & 27.7 & 7 & 23.3 \\
\hline Abdominal & 47 & 72.3 & 23 & 76.7 \\
\hline \multicolumn{5}{|l|}{ Metastases } \\
\hline Total n. cases & 39 & 60 & 15 & 50 \\
\hline Lymph nodes & 30 & 46.2 & 15 & 50 \\
\hline Bone marrow & 39 & 60 & 15 & 50 \\
\hline CNS & 1 & 1.5 & 7 & 23.3 \\
\hline Bone & 38 & 58.5 & 11 & 36.7 \\
\hline Soft tissue & 2 & 3.1 & 4 & 13.3 \\
\hline \multicolumn{5}{|l|}{ Evan stage } \\
\hline III & 26 & 40 & 15 & 50 \\
\hline IV & 39 & 60 & 15 & 50 \\
\hline
\end{tabular}

Table 2. Results of treatment of stage 3 cases.

\begin{tabular}{lccccc} 
Response & \multicolumn{2}{c}{$\begin{array}{c}\text { First-line } \\
\text { chemotherapy }\end{array}$} & \multicolumn{2}{c}{$\begin{array}{c}\text { Second-line } \\
\text { chemotherapy }\end{array}$} \\
N. & $\%$ & & N. & $\%$ \\
CR & 14 & 21.5 & 0.0064 & 6 & 20 \\
PR & 16 & 24.6 & & 18 & 60 \\
SD & 23 & 35.4 & & 2 & 6.7 \\
NR & 5 & 7.7 & 0.0064 & 1 & 3.3 \\
DP & 7 & 10.8 & & 3 & 10 \\
\hline
\end{tabular}

P value comparison between first and second groups. 
a few patients become long-term survivors. Most high-risk patients relapse after initial response to induction treatment. The optimum treatment for high-risk neuroblastoma has still not been established and the results of most of the protocols regarding, CR, event free and overall survival rates are unsatisfactory.

Our study is one of the few studies to analyze the results of conventional treatment of stage 3 and 4 neuroblastoma cases applied in the majority of cancer centers with unsatisfactory long-term outcome. It was noted that a small percentage of the cases could be cured with chemotherapy. These were cases with: low tumor burden, negative nmyc amplification, younger age at presentation, and no distant metastases.

It was observed that the majority of cases were abdominal and thoracic; this leads us to screen cases by abdomen-pelvic ultrasound and chest X-ray. Metastases were more common in bone marrow, followed by bone and lastly lymph nodes, confirming the importance of primary baseline bone marrow biopsies and isotopic bone scans for all cases. Moreover, early diagnosis is of utmost importance as it indicates better treatment outcomes, underlining the importance of screening programs, especially for high-risk groups. ${ }^{19}$

The current study showed that the initial treatment of neuroblastoma cases carries a better chance of obtaining CR that is reflected on overall survival. This indicates the importance of optimizing the primary treatment to induce the highest possible CR.

Even in cases with advanced disease, it is possible to obtain a remission rate of $70 \%$ or more with chemotherapy and surgery. ${ }^{20}$ However, the relapse rate is high among these patients, and many of them die because of the refractory recurrent tumor. ${ }^{2,3,5}$ In the current study, the majority of the chemotherapy-alone treated patients showed stationary disease (35.4\%) rather than CR (21.5\%) or PR (24.6\%).

Concerning survival, in the Spanish Neuroblastoma Group, the probability of survival with stage IV disease was 0.24 at five years. ${ }^{20}$ Moreover, Philip and colleagues ${ }^{21}$ reported an outcome of survival rate for patients given a highly intensive investigational megatherapy. The toxicity-related death rate in this group of 33 patients was $24 \%$. In the European registry, overall survival after five years was no more than $33 \%$ for stage 3 and stage 4 patients. ${ }^{22}$ The current study showed a $40 \%$ survival in the first-line chemotherapy group that is very similar to the

Table 3. Results of treatment of metastatic cases.

\begin{tabular}{lccccc} 
Response & \multicolumn{2}{c}{$\begin{array}{c}\text { First-line } \\
\text { chemotherapy }\end{array}$} & P & \multicolumn{2}{c}{$\begin{array}{c}\text { Second-line } \\
\text { chemotherapy }\end{array}$} \\
& N. & $\%$ & & N. & $\%$ \\
CR & $3 / 14$ & 21.4 & 0.0018 & $2 / 6$ & 33.3 \\
PR & $9 / 16$ & 56.3 & & $7 / 18$ & 38.9 \\
SD & $15 / 23$ & 65 & & $2 / 2$ & 100 \\
NR & $5 / 5$ & 100 & 0.0016 & $1 / 1$ & 100 \\
DP & $7 / 7$ & 100 & & $3 / 3$ & 100 \\
\hline
\end{tabular}

Pvalue comparison between first and second groups.

Table 4. Toxicity of treatment in the studied groups.

\begin{tabular}{|c|c|c|c|c|}
\hline $\begin{array}{l}\text { Toxicity } \\
\text { type }\end{array}$ & & py & $\begin{array}{l}\text { Sec } \\
\text { che } \\
\text { N. }\end{array}$ & $\begin{array}{l}\text { ne } \\
\text { rapy } \\
\%\end{array}$ \\
\hline $\begin{array}{l}\text { Grade II-III } \\
\text { myelosuppression }\end{array}$ & $39 / 65$ & 60 & $21 / 30$ & 70 \\
\hline Grade II-III vomiting & $50 / 65$ & 77 & $22 / 30$ & 73 \\
\hline Grade III alopecia & $65 / 65$ & 100 & $30 / 30$ & 100 \\
\hline Abdomenal distension & 0 & & $20 / 30$ & 66.6 \\
\hline Massive hemorrhage & 0 & & $1 / 30$ & 3 \\
\hline
\end{tabular}

Euproean study ${ }^{22}$ and that of Philip et al. ${ }^{21}$ and higher than that of the Spanish group. ${ }^{20}$ However, in the current study, survival figures were markedly lower to $14.1 \%$ at five years in the second-line chemotherapy group. This was due to toxicity build up, mainly in the form of myelosuppression, with a poor performance status and impaired vital organ reserve reducing the tolerance to chemotherapy and consequently survival. ${ }^{16,17}$

Sixty percent of neuroblastomas in young children reported by the literature are Stage 4 (undifferentiated and widely disseminated) at

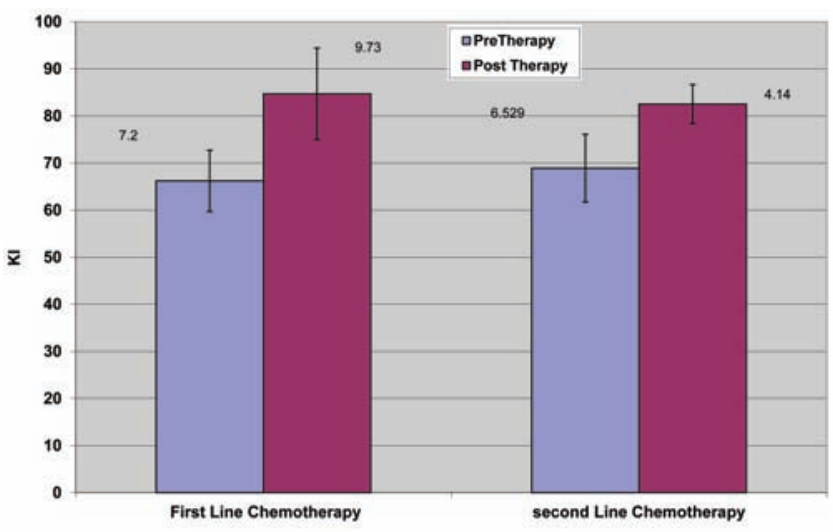

Figure 1. Comparison between the mean values of Karnofsky scale pre and post treatment.

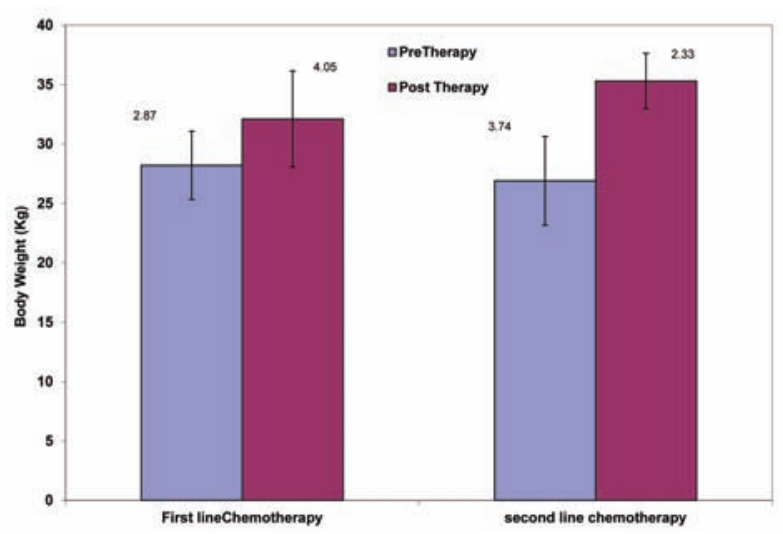

Figure 2. Comparison between the mean values of body weight pre and post treatment.

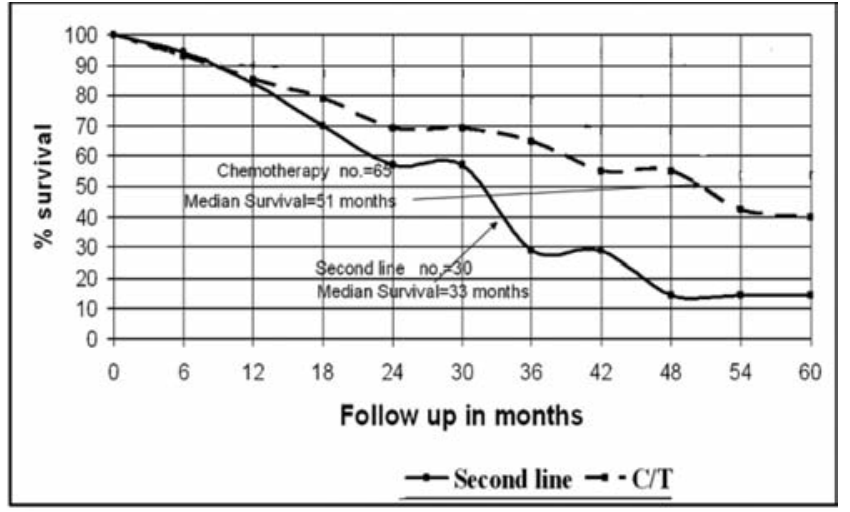

Figure 3. Comparison between total actuarial survival of the studied groups. 
diagnosis; that is very close to the percentage reported in the current study with poor treatment outcomes. ${ }^{22,23}$ Novel treatment methods are required to deal with this problem. One of the interesting new modalities is the inclusion in disease management of 131I-mIBG therapy because of the specificity of the agent and the radio-sensitivity of the primary and metastatic disease. ${ }^{24,25}$ Another investigational treatment is differentiation therapy with retinoids. Because neuroblastomas are classified as embryonal tumors arising from immature cells of the neural crest, the induced differentiation of neuroblastoma cells using retinoids either alone or in combination with other treatments has become a part of therapeutic protocols. ${ }^{26,27}$ This differentiating effect is achieved by all-trans retinoic acid (ATRA). All-trans retinoic acid is a major mediator of the effects of vitamin A via activation of a number of RAR and RXR nuclear receptors that heterodimerize and regulate gene transcription.Neuroblastomas show a high level of multidrug resistance during chemotherapy. In 2009, Choudhury and colleagues explored the effect of a combination of sorafenib and genistein on growth inhibition of neuroblastoma cells. They found that this combination abolishes the expression of MDR in both neuroblastoma SK-NDZ and SH-SY5Y cell lines. ${ }^{28}$

In an attempt to treat neuroblastoma by monoclonal antibodies, it was found that neuroblastoma cells are the tumor cells expressing the most GD2. This expression of tumor selection makes it an attractive target for tumor specific therapies, such as antibody therapy. Several anti-GD2 antibodies have been developed and used either alone or in combination with other agents in the treatment of resistant and refractory neuroblastoma. ${ }^{29-31}$ Immunotherapy may be particularly effective for low levels of minimal residual disease that is responsible for disease relapse after initial remission. Prevention of these relapses by additional conventional chemotherapy is limited because of cumulative toxicity. Thus, additional treatments to chemotherapy, surgery, and radiotherapy have to be sought. Monoclonal antibodies directed against GD2 have offered another promising avenue for treating minimal residual disease. ${ }^{32,33}$ Moreover, metronomic low-dose chemotherapy was thought to have the potential to prevent relapses with acceptably low toxicity. Therefore, an oral chemotherapy for consolidation of remission with cyclophosphamide, etoposide and melphalan was introduced in trial NB90.34,35

Finally, we would like to add that among the problems we faced during this study, was the fact that the majority of parents were unwilling to agree that their children receive any investigational therapy that had no reliable outcome. These parents, for cultural and religious reasons, would not accept any further treatment after failure of conventional therapies.

\section{Conclusions}

Even today, the treatment of advanced neuroblastoma has a very high failure rate, although some there has been some decrease in both progression and relapse rates over time. Despite this progress, neuroblastoma remains a challenging disease for both clinicians and researchers due to the lack of acceptable cure rates in high-risk neuroblastoma. Clearly, new therapeutic front-line strategies are needed to significantly increase survival. In the unfortunate case of recurrence or in the case of tumor progression, experimental therapies can be proposed. The patient's quality of life should also be carefully discussed and evaluated, and strategies for appropriate care when cure appears unlikely should receive strong consideration.

Studies using a combination of radionuclide therapy with conventional chemotherapy offer a tempting treatment option for high-risk cases of neuroblastoma, but extensive research is needed to confirm impact on survival and late effects. Future efforts should be directed to design protocols that have a multimodality treatment approach.

\section{References}

1. Berthold F, Boos J, Burdach S, et al. Myeloablative megatherapy with autologous stem-cell rescue versus oral maintenance chemotherapy as consolidation treatment in patients with highrisk neuroblastoma: a randomised controlled trial. Lancet Oncol 2005;6:649-58.

2. Katzenstein HM, Kent PM, London WB, Cohn SL. Treatment and outcome of 83 children with intraspinal neuroblastoma: the pediatric oncology group experience. J Clin Oncol 2001;19:1047-55.

3. Schmidt ML, Lukens JN, Seeger RC, et al. Biologic factors determine prognosis in infants with stage iv neuroblastoma: a prospective children's cancer group study. J Clin Oncol 2000;18:1260-8.

4. Cohn SL, Pearson AD, London WB. The International Neuroblastoma Risk Group (INRG) classification system: an INRG task force report. J Clin Oncol 2009;27:289-97.

5. Corazzari M, Lovat PE, Oliverio S, et al. Growth and DNA damageinducible transcription factor 153 mediates apoptosis in response to fenretinide but not synergy between fenretinide and chemotherapeutic drugs in neuroblastoma. Mol Pharmacol 2003;64:1370-8.

6. Lovat PE, Ranalli M, Bernassola F, et al. Synergistic induction of apoptosis of neuroblastoma by fenretinide or CD437 in combination with chemotherapeutic drugs. Int J Cancer 2000;88:977-85.

7. Saulnier-Sholler GL, Kalkunte S, Greenlaw C, et al. Antitumor activity of Nifurtimox observed in a patient with neuroblastoma. J Pediatr Hematol Oncol 2006;28:693-5.

8. Saulnier-Sholler G, Ferguson W, Brard L, et al. A phase I study of nifurtimox in patients with relapsed/refractory neuroblastoma. 44th American Society of Clinical Oncology Annual Meeting, Chicago, IL, USA, 2008.

9. Kerbel RS, Kamen BA. The anti-angiogenic basis of metronomic chemotherapy. Nat Rev Cancer 2004;4:423-36.

10. Hanahan D, Bergers G, Bergsland E. Less is more, regularly: metronomic dosing of cytotoxic drugs can target tumor angiogenesis in mice. J Clin Invest 2000;105:1045-7.

11. Shaked Y, Emmenegger U, Man S, et al. Optimal biologic dose of metronomic chemotherapy regimens is associated with maximum antiangiogenic activity. Blood 2005;106:3058-61.

12. Modak S, Cheung NK. Disialoganglioside directed immunotherapy of neuroblastoma. Cancer Invest 2007;25:67-77.

13. Simon T, Hero B, Faldum A, et al. Consolidation treatment with chimeric anti-GD2-antibody Ch14.18 in children older than 1 year with metastatic neuroblastoma. J Clin Oncol 2004;22:3549-57.

14. Navid F, Santana VM, Barfield RC. Anti-GD2 antibody therapy for GD2-expressing tumors. Curr Cancer Drug Targets 2010;10:200-9.

15. Brans B, Lindenb 0, Giammarilec F, et al. Clinical applications of newer radionuclide therapies. Eur J Cancer 2006;42:994-1003.

16. Tweedle DA, Pinkerton CR, Lewis IJ, et al. OPEC/OJEC for neuroblastoma for children over 1 year of age. Med Pediatr Oncol 2001; $36: 239-42$.

17. Rubie H, Michon J, Plantaz D, et al. Unresectable localized neuroblastoma: improved survival after primary chemotherapy including carboplatin-etoposide. Neuroblastoma Study Group of the Societe Francaise d'Oncologie Pediatrique (SFOP). Br J Cancer 1998;77: 2310-7.

18. Suita S, Zaizen Y, Sera Y, et al. Neuroblastoma in infants aged less than 6 months: is more aggressive treatment necessary? A Report from The Pediatric Oncology Study Group of the Kyushu Area. J Pediat Surg 1995;30:715-21. 
19. Woods WG, Gao RN, Shuster JJ, et al. Screening of infants and mortality due to neuroblastoma. New Eng J Med 2002;346:1041-6.

20. Klingebiel T, Bader P, Bares R, et al. Treatment of neuroblastoma stage 4 with 131i-meta-iodo-benzylguanidine, high-dose chemotherapy and immunotherapy. A pilot study. Eur J Cancer 1998;34:1398-402.

21. Philip T, Ladenstein R, Zucker JM, et al. Double megatherapy and autologous bone marrow transplantation for advanced neuroblastoma: the LMCE2 study. Br J Cancer 1993;67:119-27.

22. Philip T, Ladenstein R, Lasset C. myeloablative procedures followed by stem cell rescue for neuroblastoma: 17 years of European experience and conclusions. Eur J Cancer 1997;33:2130-5.

23. Weinstein JL, Katzenstein HM, Cohn SL. Advances in the diagnosis and treatment of neuroblastoma. Oncologist 2007;8:278-92.

24. Lashford LS, Lewis IJ, Fielding SL, et al. Phase I/II study of iodine 131 metaiodobenzylguanidine in chemoresistant neuroblastoma: a United Kingdom Children's Cancer Study Group investigation. J Clin Oncol 1992;10:1889-96.

25. Matthay KK, Tan JC, Villablanca JG, et al. Phase I dose escalation of iodine-131-metaiodobenzylguanidine with myeloablative chemotherapy and autologous stem-cell transplantation in refractory neuroblastoma: a new approaches to neuroblastoma therapy consortium study. J Clin Oncol 2006;24:500-6.

26. Reynolds CP, Matthay KK, Villablanca JG, Maurer BJ. Retinoid therapy of high-risk neuroblastoma. Cancer Lett 2003;197:185-92.

27. Sterba J, Valik D, Mudry P, et al. Combined biodifferentiating and antiangiogenic oral metronomic therapy is feasible and effective in relapsed solid tumors in children: single-center pilot study. Onkologie 2006;29:308-13.

28. Choudhury SR, Karmakar S, Banik NL, Ray SK. Synergistic effica- cy of sorafenib and genistein in growth inhibition by down regulating angiogenic and survival factors and increasing apoptosis through upregulation of p53 and p21 in malignant neuroblastoma cells having $\mathrm{N}-\mathrm{Myc}$ amplification or non-amplification. Invest New Drugs 2010;28:812-24.

29. Kushner BH, Kramer K, Cheung NK. Phase II trial of the antiG(D2) monoclonal antibody 3F8 and granulocyte-macrophage colony stimulating factor for neuroblastoma. J Clin Oncol 2001;19: 4189-94.

30. Yu AL, Batova A, Gribi R, et al. A phase III randomized trial of chimeric anti-GD2 and GMCSF/L2 in high-risk neuroblastoma following myeloablative therapy and autologous stem cell transplant (ASCT). Proc Am Soc Clin Oncol 2004;23:183.

31. Yu AL, Gilman AL, Ozkaynak MF, et al. Anti-GD2 antibody with GMCSF, interleukin-2 and isotretinoin for neuroblastoma. N Engl J Med 2010;363:1324-34.

32. Simon T, Hero B, Faldum A, et al. Consolidation treatment with chimeric anti-GD2-antibody ch14.18 in children older than 1 year with metastatic neuroblastoma. J Clin Oncol 2004;22:3549-57.

33. Simon T, Hero B, Faldum A, et al. Infants with stage 4 neuroblastoma: the impact of the chimeric anti-GD2-antibody ch14.18 consolidation therapy. Klin Padiatr 2005;217:147-52.

34. Andre N, Pasquier E, Verschuur A, et al. Metronomic chemotherapy in pediatric oncology: hype or hope? Arch Pediatr 2009;16:115865.

35. Stempak D, Seely D, Baruchel S. Metronomic dosing of chemotherapy: Applications in pediatric oncology. Cancer Invest 2006;24:43243. 